\title{
A Min-Max theorem about the Road Coloring Conjecture
}

\author{
Rajneesh Hegde ${ }^{1 \dagger}$ and Kamal Jain ${ }^{2 \ddagger}$ \\ ${ }^{1}$ Georgia Institute of Technology, Atlanta, GA 30332, USA. \\ ${ }^{2}$ Microsoft Research Center, Redmond, WA 98052, USA.
}

The Road Coloring Conjecture is an old and classical conjecture posed in Adler and Weiss (1970); Adler et al. (1977). Let $G$ be a strongly connected digraph with uniform out-degree 2 . The Road Coloring Conjecture states that, under a natural (necessary) condition that $G$ is "aperiodic", the edges of $G$ can be colored red and blue such that "universal driving directions" can be given for each vertex. More precisely, each vertex has one red and one blue edge leaving it, and for any vertex $v$ there exists a sequence $s_{v}$ of reds and blues such that following the sequence from any starting vertex in $G$ ends precisely at the vertex $v$. We first generalize the conjecture to a min-max conjecture for all strongly connected digraphs. We then generalize the notion of coloring itself. Instead of assigning exactly one color to each edge we allow multiple colors to each edge. Under this relaxed notion of coloring we prove our generalized Min-Max theorem. Using the Prime Number Theorem (PNT) we further show that the number of colors needed for each edge is bounded above by $O(\log n / \log \log n)$, where $n$ is the number of vertices in the digraph.

Keywords: road coloring, synchronization of automata

\section{Introduction}

Imagine a network of one-way roads between a set of cities, such that there are exactly two roads leaving each city. The road coloring problem asks when it is possible to color the roads red and blue such that every city can be assigned universal driving directions. In other words, for every destination city, there is a sequence of reds and blues such that following the entire sequence from any starting city ends precisely in the destination city. (Note that the sequence must end on the city, not just pass through it.) For such a coloring to exist, two necessary conditions are (i) the network is strongly connected, and (ii) it is "aperiodic" (see Section 2). The Road Coloring Conjecture states that these necessary conditions are sufficient too.

The problem arises naturally in symbolic dynamics Adler et al. (1977); Béal and Perrin (1997) and has applications in automata theory Perrin and Schützenberger (1992). In the context of an automaton, a synchronizing sequence as above makes it resistant to input errors, since the sequence can be used to reset the automaton back to the required state no matter where the error occurred.

\footnotetext{
†rdhemath.gatech.com

¥kamalj@microsoft.com
} 
We extend the conjecture to digraphs that are not necessarily aperiodic. This casts the traditional Road Coloring Conjecture in an elegant min-max form. We prove this conjecture under an additional flexibility that a road can be assigned more than one color. For instance, the same road can be called Highway 95 as well as New Jersey Turnpike. Our theorem holds for all strongly connected digraphs, irrespective of out-degree. Our theorem uses at most $O(\log n / \log \log n)$ colors on any edge, where $n$ is the number of vertices in the digraph. We give an example where multiple colors are needed for an edge. We suspect that the asymptotic bound above is tight. The extended road coloring conjecture requires assigning exactly one color to every edge, provided the out-degrees are uniform.

\section{Problem Definitions}

Traditional Road Coloring Conjecture (T-RCC): We are given a strongly connected digraph, $G=$ $(V, E)$. Each vertex has uniform out-degree $\Delta$. We are also given a set of $\Delta$ different colors. Let us denote the set of colors by $C_{\Delta}=\left\{c_{1}, c_{2}, \ldots, c_{\Delta}\right\}$. A proper coloring of edges is an assignment of one color to every edge such that no two edges leaving a vertex get the same color. Throughout this paper, all colorings considered will be proper. Given a coloring of the edges we can define a function $f_{s}: V \rightarrow V$ for every finite sequence $s$ of colors. We call this function the traveling function for the sequence $s$. For every vertex $v, f_{s}(v)$ is the ending vertex if we follow the sequence $s$ starting at $v$. A coloring is said to be synchronizing if there exists a sequence $s$ such that $\left|f_{s}(V)\right|=1$. In other words, following $s$ will lead to the unique vertex $v$ in the range of $f_{s}$, irrespective of where we started. Such a sequence $s$ is called a synchronizing sequence for $v$, or simply a synchronizing sequence. Clearly, if $G$ is strongly connected, then it has a synchronizing sequence for some vertex if and only if it has one for every vertex. (If $s$ is a synchronizing sequence for $v$, then for any other vertex $w$, simply pick any path from $v$ to $w$ and append the sequence of colors on this path to $s$ to obtain a synchronizing sequence for $w$.)

$G$ is said to have a cyclic $k$-partition if the vertex set of $G$ can be partitioned into $k$ parts $V_{0}, V_{1}, V_{2}, \ldots$, $V_{k-1}$ such that every edge goes from one part to the next, i.e., if $(u, v)$ is an edge and $u \in V_{i}$, then $v \in V_{(i+1)} \bmod k$. Clearly, if $G$ has a cyclic $k$-partition for $k>1$, then it cannot have a synchronizing coloring. $G$ is said to be aperiodic if it does not admit a cyclic $k$-partition for any $k>1$.

Conjecture 1 (T-RCC) Every strongly connected, aperiodic digraph with uniform out-degree $\Delta$ has a synchronizing coloring with $\Delta$ colors.

The simplest case of T-RCC is when every vertex has uniform out-degree 2. Even this case has not been settled completely yet. However, the conjecture has been proved under various special conditions. For instance, Kari (2003) prove T-RCC for Eulerian digraphs; Jonoska and Suen (1995) prove T-RCC when the digraph can be colored such that each color class is connected (i.e. it contains exactly one cycle) and the gcd of those cycle lengths is one. For other special cases, see O'Brien (1981); Friedman (1990); Carbone (2001); Perrin and Schützenberger (1992).

Strong Road Coloring Conjecture (S-RCC): T-RCC allows only aperiodic digraphs. We wish to relax the condition of aperiodicity. As mentioned above, if a digraph is not aperiodic then it does not admit any synchronizing coloring. So we need to extend the notion of synchronization itself. Let us first extend the notion of aperiodicity and then the notion of synchronization.

If a digraph $G$ admits a cyclic $k$-partition for some $k>1$ then the digraph is said to be periodic. The largest $k$ for which it admits a $k$-partition is called its period. Aperiodic digraphs have period one.

We define the synchronizing number of a coloring for a digraph $G$ as the minimum of $\left|f_{s}(V)\right|$ over all 
the sequences of colors $s$. The synchronizing number of a digraph $G$ is the minimum of synchronizing number over all proper colorings of $G$.

Note that the period of a digraph is an obvious lower bound on its synchronizing number. Clearly, if $G$ admits a cyclic $k$-partition then the synchronizing number of any coloring has to be at least $k$, that is, for any $s,\left|f_{s}(V)\right| \geq k$. Indeed, $f_{s}(V)$ will have at least one vertex from each part of the partition. A vertex from $V_{i}$ is in $f_{s}(V)$ because $f_{s}\left(V_{(i-|s|}\right)$ mod $\left.k\right)$ is in $V_{i}$. This leads to the following min-max conjecture:

Conjecture 2 (S-RCC) For every strongly connected digraph $G$ with uniform out-degrees, the period of $G$ is equal to its synchronizing number.

Clearly T-RCC is a special case of S-RCC, when the digraphs are restricted to aperiodic digraphs.

S-RCC can be proved under the additional condition that $G$ is Eulerian, using an adaptation of the proof in Kari (2003).

Theorem 1 For every strongly connected, Eulerian digraph $G$ with uniform out-degrees, the period of $G$ is equal to its synchronizing number.

Multicolor Road Coloring Theorem (mRCT): In this paper we prove a road coloring theorem which extends the S-RCC to all strongly connected digraphs under a suitably extended notion of coloring. Validity of S-RCC is restricted to uniform out-degree digraphs only. We allow digraphs with non-uniform out-degrees too. In this case the notion of proper coloring must include one of the following possibilities: (i) an edge gets more than one color, (ii) an edge gets no color, (iii) a vertex misses some color on any of its outgoing edges, or (iv) a vertex sees the same color on more than one of its outgoing edge.

Let us discuss these four possibilities and see how they affect the notion of synchronization. If we allow (iv) above, then there is a confusion in case we have to follow the duplicated color from the vertex. In this case either (a) we have to guarantee that the confusion is not faced by carefully choosing the synchronizing sequence or (b) we have to allow the function $f_{s}$ to carryover this confusion, i.e., $f_{s}$ of a vertex need not be a single vertex but a set of vertices. If we follow (a) then (iv) is reduced to (iii), which we will discuss later. Following (b) is a consistent scenario but we do not pursue this in this paper. The fact that the period is a lower bound on the synchronizing number remains valid. So it is a natural question to ask whether this lower bound is tight after one extends the notion of coloring under (iv-b).

If we allow (iii) then we have to guarantee that our synchronizing sequence does not ask to follow the missing color from that vertex. Again, the fact that the period is a lower bound on the synchronizing number remains valid. The following counterexample shows that this lower bound is not tight unless we also allow (i). Consider the digraph on the vertex set $\{u, v, w\}$ with edges $(u, v),(u, w),(w, v)$ and $(v, u)$. The period of this digraph is one, and it is easy to show that if we only allow (iii), then the synchronizing number cannot be one.

If we allow (ii) then it is equivalent to removing an edge. If removing an edge does not change the period then this edge can be removed without changing the validity of the tightness of the lower bound. In case of minimal counterexample, we can assume that removal of any edge changes the period. This change can only increase the period. There are digraphs with non-uniform out-degrees in which removal of any edge increases the period, e.g. the digraph above. So if we do not give a color to any edge then the lower bound can't be tight unless we allow (i) also.

So in this paper we allow (i). Since allowing (ii) comes for free with allowing (i) (we can always put dummy colors on edges and not use them in our synchronizing word), we allow (ii) also. We show that if we allow the possibility of multiple colors to every edge then the min-max relation given by S-RCC is true. We further prove that the number of colors on each edge is at most $O(\log n / \log \log n)$, where $n$ is 
the number of vertices. In fact, since we are allowing (ii), the number of colors we use itself is at most $O(\log n / \log \log n)$. The above example shows that multiple colors are required. We further conjecture that asymptotically our bound is tight.

The preceding discussion thus leads to the following notion of a proper multi-coloring: an assignment of zero, one or more colors to every edge of a digraph $G$ such that no two edges leaving a vertex receive a common color.

\section{Theorem Statements and Proof Ideas}

The core of our idea is the use of the notion of jellyfish. (The notion is also used in Jonoska and Suen (1995) and Carbone (2001); the latter refers to it as a "complete C-cover".) A jellyfish is a connected digraph (in an undirected sense; not necessarily strongly connected) with uniform out-degree one. Clearly such a digraph has exactly one cycle. The nodes on this cycle are reachable from any vertex in the digraph. As usual, a subgraph of $G$ is said to be spanning if its vertex set is the same as that of $G$.

The following lemma is trivial to prove.

Lemma 2 Given a strongly connected digraph $G$ and any cycle in it, we can choose a spanning subgraph of $G$ that is a jellyfish with the given cycle.

The following well-known observation gives an alternate characterization of the period.

Lemma 3 For any digraph $G$ and any positive integer $k, G$ admits a cyclic $k$-partition if and only if $k$ divides the length of every (directed) cycle in $G$. Further, if $G$ admits a cyclic $k$-partition, then the partition is unique up to cyclic permutation of the parts $V_{0}, \ldots, V_{k-1}$.

Corollary 3.1 The period of a digraph $G$ is the gcd of the lengths of the (directed) cycles of $G$.

The following lemma follows from Lemma 2 and Corollary 3.1.

Lemma 4 Given a digraph, $G$, we can find a set of spanning subgraphs of $G$ that are all jellyfishes, such that the gcd of the cycle lengths of these jellyfishes is the period of $G$.

We pick such a set of jellyfishes and for each one of them, we give one separate color to all its edges. Note that these jellyfishes were not necessarily edge-disjoint so some edges of the digraph may get more than one color. Also note that these jellyfishes need not cover all the edges of the digraph so some edges may not get any color. The number of colors we are using is the number of jellyfishes we have. We will show that it is possible to pick a set of $O(\log n / \log \log n)$ jellyfishes to realize the period of $G$. This will prove the required upper bound on the number of colors we assign to any edge.

The proof in Jonoska and Suen (1995) can be adapted to prove the following main lemma.

Lemma 5 Given a digraph $G$ and a proper multi-coloring of it such that some color class induces a jellyfish (i.e. induces a connected subgraph), then the synchronizing number of the coloring divides the cycle length of that jellyfish.

From Lemmas 4 and 5, we get a proper multi-coloring of $G$ whose synchronizing number divides the period of $G$. However, since the synchronizing number cannot be smaller than the period of the graph, we get the following theorem.

Theorem 6 For any strongly connected digraph $G$, the period of $G$ is equal to its synchronizing number. 
Now we show that given a digraph with $n$ vertices, its period can be realized as the gcd of at most $O(\log n / \log \log n)$ cycles. Let the period of the given digraph be $k$. Suppose the minimum number of cycles we need to realize the period is $t$. Let the cycle lengths of these $t$ cycles be $l_{1}^{\prime}, l_{2}^{\prime}, \ldots, l_{t}^{\prime}$ respectively. The gcd of these cycle lengths is $k$. Let us divide all the cycle lengths by $k$, say $l_{i}=l_{i}^{\prime} / k$. Now the gcd of $l_{1}, l_{2}, \ldots l_{t}$ is 1 .

Since we chose the minimum number of cycles to realize the gcd, for any $1 \leq i \leq t$, the gcd of the numbers $\left\{l_{j}: j \neq i\right\}$ is strictly more than one. Thus there exists a prime number, say $p_{i}$, which divides $l_{j}$ for every $j \neq i$ but does not divide $l_{i}$. Clearly, for $i \neq j$, we have $p_{i} \leq p_{j}$. Thus each $l_{i}$ is at least a product of $t-1$ distinct primes. In particular, each $l_{i}$ is at least the product of the first $t-1$ primes. Since $n$ is at least the maximum of the $l_{i}$ 's, $n$ is at least the product of the first $t-1$ primes.

Let us say that the $(t-1)^{\text {th }}$ prime is $p$. From the prime number theorem (PNT) we can deduce that asymptotically half of the first $t$ primes are of magnitude $\Theta(p)$. So $n$ is at least $(\Theta(p))^{t / 2}$. Again from the PNT $t$ is $\Theta(p / \log p)$. Eliminating $t$ and taking $\operatorname{logarithms~shows~that~} \log n$ is at least $\Theta(p)$. Eliminating $p$ gives us that $t$ is at most $\Theta(\log n / \log \log n)$, that is, $t$ is $O(\log n / \log \log n)$.

Theorem 7 For any strongly connected digraph $G$, the period of $G$ is equal to the synchronizing number of $G$. If $G$ has $n$ vertices then $O(\log n / \log \log n)$ colors are sufficient to achieve this synchronization.

\section{Discussion}

It is easy to prove, using a pigeon-hole principle argument, that given a strongly connected digraph and a proper multi-coloring of its edges, there exists a sequence of length at most $n^{3}$ that achieves its synchronizing number. (A related question is Cěrný's conjecture, which states that if a coloring is synchronizing, then there exists a synchronizing sequence of length at most $(n-1)^{2}-$ see Cěrný (1964); Pin (1983).) The above argument also gives a trivial $O\left(n^{4}\right)$-time algorithm to compute the synchronizing number for a coloring (and in particular, to determine whether it is equal to the period of the digraph). However, we do not know any good structural characterization of when a coloring has synchronizing number equal to the period. It is likely that finding such a characterization would shed some light on how to prove the strong Road Coloring Conjecture.

\section{References}

R. L. Adler, L. W. Goodwyn, and B. Weiss. Equivalence of topological Markov shifts. Israel J. Math., 27 (1):48-63, 1977. ISSN 0021-2172.

R. L. Adler and B. Weiss. Similarity of automorphisms of the torus. Memoirs of the American Mathematical Society, No. 98. American Mathematical Society, Providence, R.I., 1970.

M.-P. Béal and D. Perrin. Symbolic dynamics and finite automata. In Handbook of formal languages, Vol. 2, pages 463-505. Springer, Berlin, 1997.

A. Carbone. Cycles of relatively prime length and the road coloring problem. Israel J. Math., 123: 303-316, 2001. ISSN 0021-2172.

J. Cěrný. Poznámka k homogennym experimenton s konečnými automatmi. Mat. fyz. čas. SAV, 14: 208-215, 1964. 
K. Culik, II, J. Karhumäki, and J. Kari. A note on synchronized automata and road coloring problem. Internat. J. Found. Comput. Sci., 13(3):459-471, 2002. ISSN 0129-0541.

J. Friedman. On the road coloring problem. Proc. Amer. Math. Soc., 110(4):1133-1135, 1990. ISSN 0002-9939.

G. J. O. Jameson. The Prime Number Theorem. Cambridge University Press, 2003. ISBN 0521891108.

N. Jonoska and S. Suen. Monocyclic decomposition of graphs and the road coloring problem. In Proceedings of the Twenty-sixth Southeastern International Conference on Combinatorics, Graph Theory and Computing (Boca Raton, FL, 1995), volume 110, pages 201-209, 1995.

J. Kari. Synchronizing finite automata on Eulerian digraphs. Theoret. Comput. Sci., 295(1-3):223-232, 2003. ISSN 0304-3975. Mathematical foundations of computer science (Mariánské Lázně, 2001).

G. L. O'Brien. The road-colouring problem. Israel J. Math., 39(1-2):145-154, 1981. ISSN 0021-2172.

D. Perrin and M.-P. Schützenberger. Synchronizing prefix codes and automata and the road coloring problem. In Symbolic dynamics and its applications (New Haven, CT, 1991), volume 135 of Contemp. Math., pages 295-318. Amer. Math. Soc., Providence, RI, 1992.

J.-E. Pin. On two combinatorial problems arising from automata theory. In Combinatorial mathematics (Marseille-Luminy, 1981), volume 75 of North-Holland Math. Stud., pages 535-548. North-Holland, Amsterdam, 1983. 